Fisioter Bras 2021;22(2):154-67

doi: $10.33233 / f b . v 22 i 2.4553$

\title{
ARTIGO ORIGINAL \\ Uso da eletromiografia de superfície na resultância do treinamento muscular inspiratório com carga linear em portadores do vírus linfotrópico humano tipo 1 Use of surface electromyography in results of inspiratory muscle training with linear load in patients with type 1 human lymphotropic virus
}

Amanda Caroline Lobato Dias*, Kayonne Campos Bittencourt, Ft., D.Sc. ${ }^{*}$, Isabelle Farias Gomes*, Denise da Silva Pinto, Ft., D.Sc. **, Luiz Fábio Magno Falcão, Ft., D.Sc. **, João Sérgio de Sousa Oliveira, Ft., D.Sc.**, Valéria Marques Ferreira Normando, Ft., D.Sc. **

*Estudantes de Fisioterapia da Universidade do Estado do Pará (UEPA), Belém, PA, **Professor Universidade do Estado do Pará (UEPA), Belém, PA

Recebido em 6 de janeiro de 2021; aceito em 16 de abril de 2021.

Correspondencia: Amanda Caroline Lobato Dias, Tv. Perebebuí, 2623 Marco 66087662 Belém, PA, Brasil

Amanda Caroline Lobato Dias: amandacarolineld11@gmail.com Kayonne Campos Bittencourt: kayonnebitten@gmail.com Isabelle Farias Gomes: isabelleffisio@gmail.com Denise da Silva Pinto: denisepinto@ufpa.br Luiz Fábio Magno Falcão: fabiofisiofalcao@yahoo.com.br João Sérgio de Sousa Oliveira:joaosergio@uepa.br Valéria Marques Ferreira Normando: valeriafisio@gmail.com

\section{Resumo}

Este estudo visa avaliar por eletromiografia de superfície o comportamento dos músculos inspiratórios no treinamento muscular em voluntários com vírus linfotrópico de célula T humana do tipo 1. Trata-se de um ensaio clínico. Sete voluntários, com idade $58,85 \pm 7,2)$ anos, realizaram treinamento muscular inspiratório domiciliar por 4 semanas, 3 vezes por semana, 30 minutos diários por meio de incentivador de carga linear. Para avaliação utilizou-se os dados de pressão inspiratória máxima e os dados da eletromiografia de superfície nas fases pré (T0), segunda semana (T2) e após a quarta semana (T4) de treinamento. Observou-se aumento progressivo da força 
muscular inspiratória de T0 a T4 $(p=0,007)$, assim como, aumento do recrutamento das unidades motoras pela análise da amplitude do sinal eletromiográfico, sendo mais evidente para o músculo esternocleidomastóideo $(p=0,12)$ em comparação ao músculo diafragma $(p=0,6)$. Verificou-se que no decurso do treinamento muscular ocorreu melhora significativa da força muscular inspiratória com maior recrutamento das fibras musculares dos músculos analisados na amostra.

Palavras-chave: vírus linfotrópico de célula T humana do tipo 1; músculos respiratórios; eletromiografia.

\section{Abstract}

This study aimed to evaluate by surface electromyography the behavior of inspiratory muscles in the muscle training of volunteers with human T-cell lymphotropic virus type 1. This was a clinical trial. Seven volunteers, $58,85 \pm 7.21$ years old, underwent inspiratory muscle training at home for 4 weeks, 3 times a week, 30 minutes daily by means of a linear load stimulator. The maximum inspiratory pressure data and the surface electromyography data were used for evaluation in the pre (T0), second week (T2) and after the fourth week (T4) training phases. There was a progressive increase in inspiratory muscle strength from T0 to T4 $(p=0.007)$, as well as an increase in the recruitment of motor units by analyzing the amplitude of the electromyographic signal, being more evident for the sternocleidomastoid muscle $(p=0.12)$ in comparison to the diaphragm muscle $(p=0.6)$. During the muscle training inspiratory muscle strength improves with greater recruitment of muscle fibers from the muscles analyzed in the sample.

Keywords: Human T-cell lymphotropic virus type 1; respiratory muscles; electromyography.

\section{Introdução}

O vírus linfotrópico de célula T humana, HTLV (Human T Lymphotrophic Viruse) tipo 1 (HTLV-1) é um retrovírus associado a patologias como leucemia/linfoma de células $T$ do adulto (LLCTA), paraparesia espástica tropical/mielopatia associada ao HTLV-1 (PET/MAH), distúrbios inflamatórios oftalmológicos, dermatológicos, reumatológicos e osteomioarticulares não malignos [1].

Caribe, América do Sul, Ilhas da Melanésia, Oeste Africano e Japão são países prevalentes ao vírus. No Brasil, estima-se que existam cerca de 2,5 milhões de pessoas infectadas, há prevalências mais elevadas nos estados da Bahia, Pernambuco e Pará. As formas de transmissão são por via sexual, via sanguínea por meio de transfusões, 
por transplante de órgãos ou pelo uso de fômites, por via vertical de mãe para filho, pela passagem de linfócitos infectados pelo leite materno e no período perinatal [2].

Na maioria dos casos o vírus persiste de forma assintomática e indetectável pelo sistema imune, por anos ou até a morte. Quando sintomático, o indivíduo é acometido por infecção crônica que pode estar associada a doenças incapacitantes, que comprometem a qualidade de vida, como a doença neurológica PET-MAH, o qual por estímulos de linfócitos, atravessam a barreira hematoencefálica com formação de quimiocinas e citocinas pró-inflamatórias neurotóxicas [3].

Desta forma, destaca-se a possibilidade do surgimento de alterações morfológicas e funcionais também no sistema respiratório, no qual decorre de um processo inflamatório ocasionado pelo vírus com infiltração linfocítica e liberação de citocinas lesivas ao tecido pulmonar [4].

Atenta-se para alveolíte $\mathrm{T}$ linfocítica, pneumonia interesticial, bronquiolite e panbronquiolite difusa, risco aumentado para cryptococcose pulmonar, tuberculose pulmonar e pneumonia adquirida na comunidade, patologias que podem ser percebidas em portadores assintomáticos e em pacientes com PET/MAH $[5,6]$.

Segundo Falcão [7], os dados obtidos apontam que o pulmão é um sítio preferencial para o HTLV-1 e que este tropismo possa ser o causador das lesões pulmonares. A sintomatologia pulmonar é subclínica, em tal caso são descobertas ocasionalmente ou diante de sintomatologia grave, sendo esta menos comum e restrita a alguns casos, requisitando, assim, internações hospitalares.

$\mathrm{Na}$ qualidade das possibilidades terapêuticas preventivas, o treinamento muscular respiratório (TMR) surge e reflete diretamente na funcionalidade do paciente, tendo como diretriz a condução de protocolos de treinamento [8]

Como ferramenta para avaliar as atividades elétricas musculares durante o TMR, a eletromiografia de superfície (EMGs) se destaca como método não invasivo, refletindo a soma da atividade elétrica de todas as fibras musculares esqueléticas ativas [9]. Essa técnica auxilia a análise da relação entre a sincronização, o tempo de ativação dos músculos, a relação do sinal de força e o uso do sinal eletromiográfico como um índice de fadiga [10,11].

Dessa forma, este estudo teve como objetivo avaliar o comportamento eletromiográfico de músculos inspiratórios na proposta do treinamento muscular em voluntários com vírus linfotrópico de célula T humana do tipo 1. 


\section{Métodos}

Aspectos éticos

Trata-se de um estudo tipo ensaio clínico, com abordagem quantitativa, disponível em https://clinicaltrials.gov com ID NCT03829709 e com aprovação do Comitê de Ética e Pesquisa com Seres Humanos - UEPA (parecer no. 2.695.505), realizado no período de janeiro a agosto de 2019, na Unidade de Ensino e Assistência em Fisioterapia e Terapia Ocupacional (UEAFTO) da Universidade do Estado do Pará (UEPA), com voluntários infectados pelo HTLV-1 que assinaram o Termo de Consentimento Livre e Esclarecido (TCLE).

\section{Amostra}

A amostra foi estabelecida segundo os seguintes critérios de inclusão: indivíduos infectados exclusivamente pelo HTLV-1, de ambos os sexos, com faixa etária entre 50 e 70 anos, cadastrados no Laboratório de Estudo em Reabilitação Funcional (LAERF) localizado no Núcleo de Medicina Tropical da Universidade Federal do Pará. Foram excluídos indivíduos com outros acometimentos pulmonares, comorbidades como: cardiopatia e hipertensão arterial não controlada e alterações cognitivas. (Figura 1)

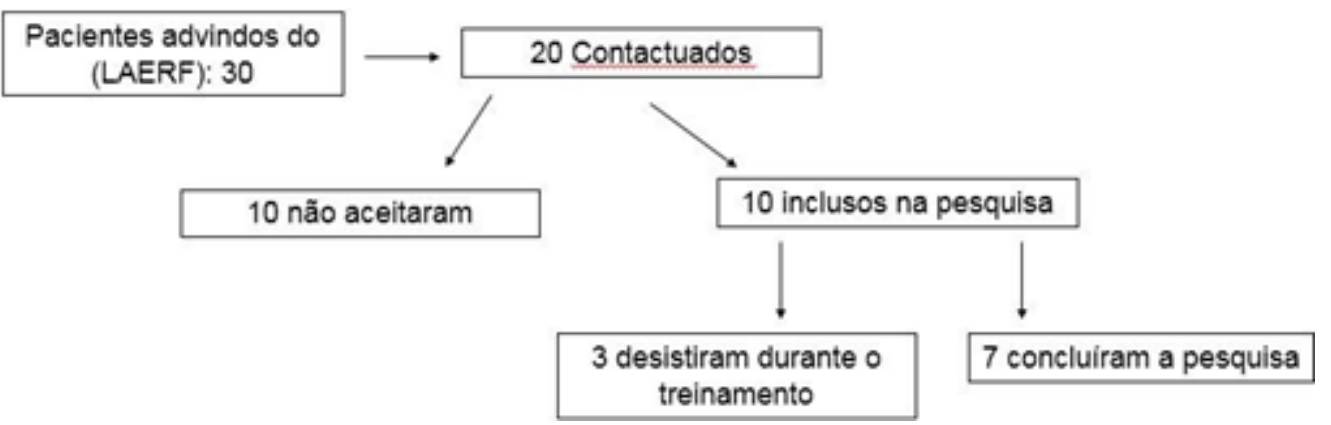

Figura 1 - Fluxograma do encaminhamento inclusivo de portadores do vírus HTLV-1 no estudo. Belém, 2019

Protocolo de avaliação

Os dados foram coletados em formulário-padrão, incluindo: dados pessoais e clínicos, além da força muscular inspiratória e da eletromiografia de superfície. 
Força muscular inspiratória

A leitura da pressão inspiratória máxima $\left(\mathrm{PI}_{\text {máx }}\right)$ foi conduzida por meio do manovacuômetro, modelo Digital MVD 300 (GlobalMed ${ }^{\mathrm{TM}}$, Porto Alegre/RS, Brasil), adotando valores teóricos previstos por Neder et al. [12].

Para tal, os voluntários foram posicionados em sedestação na cadeira com encosto, com o bocal na posição intraoral e vedação da cavidade nasal por meio de um clipe. A partir do comando verbal, o voluntário gerou o esforço inspiratório até a capacidade pulmonar total (CPT), a partir do volume residual (VR), para o registro da medida. As técnicas de mensuração foram repetidas até completarem três medidas satisfatórias da $\mathrm{PI}_{\text {máx }}$ com variação de até $10 \%$ entre estas, com intervalo de repouso de 1 minuto.

\section{Eletromiografia de superfície}

Foi utilizado o eletromiógrafo modelo Miotool 400 (Miotec®), Porto Alegre/RS, Brasil), com conversor analógico-digital de quatro canais. Para processamento dos dados foi usado o software Miograph versão MiotecSuite1.0.1108 (Miotec®, Porto Alegre/RS, Brasil), com resolução de 16 bits.

Os parâmetros do equipamento foram ajustados para um ganho final de 1000 vezes, com filtros gerando uma banda passante entre 10 e $500 \mathrm{~Hz}$ (passa-alta de $10 \mathrm{~Hz}$ e passa baixa de $500 \mathrm{~Hz}$ ), filtro Notch sintonizado para $60 \mathrm{~Hz}$ para redução da amplitude gerada pela eletricidade da rede ambiente [13].

Os sinais do equipamento utilizaram frequência de amostragem de 2.000 amostras/segundo por canal e captados por sensores diferenciais de superfície (SDS500). Utilizando eletrodos de superfície de prata cloreto de prata (Ag/AgC) com adesivos de hidrogel condutor da marca (KendalTM, Mansfield - EUA), com formato circular e diâmetros de $10 \mathrm{~mm}$, posicionados em pares com distância entre os eletrodos de $14 \mathrm{~mm}$. Antes da colocação dos eletrodos, a pele foi tricotomizada com lâmina descartável e limpa com álcool etílico $70 \%$. O procedimento de preparo e as orientações do paciente seguiram as recomendações da Surface Electromyography for the NonInvasive Assessment of Muscles (SENIAM) [14].

Os posicionamentos dos eletrodos nos músculos seguiram as recomendações padronizadas de Wu et al. [15] modificado, os quais foram posicionados no hemicorpo direito. Para o músculo diafragma, utilizou-se o primeiro ponto de intersecção no sexto espaço intercostal e o segundo no oitavo espaço intercostal. Para o músculo esternocleidomastóideo, os eletrodos foram posicionados em 1/3 e 2/3 do comprimento 
total do músculo. O eletrodo de referência foi colocado no processo estilóide da ulna do lado contralateral aos eletrodos de superfície.

A coleta dos dados eletromiográficos foi realizada a cada semana de avaliação em dois momentos, o primeiro momento, paciente sentado em uma posição confortável, ocorreu durante a verificação da $\mathrm{PI}_{\text {máx }} \mathrm{e}$ a obtenção do recrutamento das unidades motoras por meio da amplitude do sinal eletromiográfico pela expressão da raiz quadrada da média dos quadrados (RMS). No segundo momento, foi solicitado que o voluntário realizasse uma inspiração (contração isométrica) com duração de 10 segundos, por meio do dispositivo linear respiratório com a carga determinada da semana.

A normalização dos valores eletromiográficos da RMS foi direcionada para ações musculares isométricas, sendo disponibilizada em \% contração voluntária máxima (CVM), obtidas dos músculos diafragma e esternocleidomastóideo. O valor final foi normalizado por meio de variáveis quantitativas contínuas expressas em valores percentuais. Sendo associado há um maior percentual de atividade elétrica do músculo, um maior recrutamento de unidades motoras, porém com menor eficiência para o desempenho biomecânico para uma tarefa determinada.

\section{Protocolo terapêutico}

O treinamento muscular inspiratório (TMI) foi realizado 3 vezes na semana, sendo que 1 sessão monitorada pelo pesquisador na UEAFTO e 2 sessões realizadas em domicílio, por 30 minutos, ao longo de 4 semanas com base no protocolo de Ray et al. [16] modificado.

Os indivíduos receberam o dispositivo de carga linear Threshold® IMT, e as orientações devidas quanto ao manuseio, postura e assepsia. Realizaram o treinamento em sedestação com o dorso no encosto e os cotovelos apoiados em um anteparo. Foram solicitados a utilizar o clipe nasal e inspirar completamente contra uma resistência pré-ajustada pelos pesquisadores e expirar dentro do aparelho sem resistência, em seguida retiravam o aparelho e continuavam a respirar normalmente até o próximo ciclo cronometrado. Cada ciclo durou 10 segundos com descanso de 30 segundos, durante o treinamento contabilizou-se 45 incursões respiratórias com ritmo tranquilo para evitar tontura ou enjoo.

A carga de treinamento inicial para cada participante foi ajustada em $25 \%$ da PImáx. Os voluntários foram treinados e instruídos a conduzir o programa de exercícios por conta própria no seu domicílio sob monitoração à distância pelos pesquisadores. Uma vez por semana, presencialmente na UEAFTO, o pesquisador determinava os 
novos valores para carga a partir do novo registro da $\mathrm{Pi}_{\text {máx. }}$ Para a 1a avaliação $25 \%$; 2a. avaliação 35\%; 3a. avaliação 40\%; 4a. avaliação 45\%; 5a . avaliação $50 \%$. O dispositivo foi coberto com material isolante para que os participantes não tivessem acesso a mudança na carga de treinamento.

\section{Análise estatística}

Os dados coletados foram organizados em planilhas Excel 2007, submetidas à análise estatística por meio do programa Bioestat $5.0 \AA$, para verificação da normalidade adotou-se o teste de Shapiro-Wilk, para inferência estatística dos dados paramétricos foram utilizados o teste T Student ou análise de variância (ANOVA) e para inferência dos dados não paramétricos o teste de Wilcoxon. Adotou-se nível alfa de significância de $5 \%$.

\section{Resultados}

Dos sete voluntários portadores do HTLV-1 envolvidos na pesquisa, 5 (71,42\%) eram do sexo feminino e $2(28,57 \%)$ do sexo masculino, com idade 58,85 $\pm 7,21$ anos. Quanto à forma clínica, 2 (28,57\%) eram PET-MAH provável, 2 (28,57\%) PET-MAH definitivo e 3 (42,85\%), sem PET-MAH (Tabela I).

Tabela I - Perfil dos sete portadores do HTLV-1 inseridos no estudo Belém, Pará, 2019

\begin{tabular}{llll}
\hline Variáveis & N & $\%$ & Média \\
\hline Sexo & 5 & 71,42 & \\
Feminino & 2 & 28,57 & \\
Masculino & & & 58,85 \\
\hline Idade & & & \\
\hline Forma clínica & 28,57 & \\
PET-MAH provável & 2 & 28,57 & \\
PET-MAH definitivo & 2 & 42,85 & \\
SemPET-MAH & 3 &
\end{tabular}

Fonte: Protocolo de pesquisa, 2019

A Tabela II aponta os valores obtidos na EMGs realizada na avaliação inicial (T0); segunda semana (T2) e quarta semana (T4). Observa-se redução na média do potencial eletromiográfico para os músculos avaliados. Destacando expressividade para o músculo esternocleidomastóideo $(p=0,004)$, referindo um menor recrutamento de fibras musculares esqueléticas após duas semanas de treinamento, frente ao músculo diafragma $(p=0,08)$.

Diante da carga acima $40 \%$ da Pimáx, ou seja, de T2 a T4, verificou-se o aumento no potencial eletromiográfico, principalmente do músculo 
esternocleidomastóideo ( $p=0,06)$. Referente a avaliação inicial (T0) e a final (T4) ocorreu um aumento maior do recrutamento das unidades motoras, sendo mais evidente para o músculo esternocleidomastóideo $(p=0,12)$ em comparação ao músculo diafragma $(p=0,6)$.

Tabela II - Análise da eletromiografia dos músculos esternocleidomastóideo e diafragma em sete portadores de HTLV-1 participes do estudo. Belém, Pará, 2019

\begin{tabular}{llllllllll}
\hline Variável EMGs & T0 & T2 & P & T0 & T4 & P & T2 & T4 & p \\
\hline Estemocleidomastóideo & 28,86 & 26,75 & $0,004^{*}$ & 28,86 & 60,70 & 0,12 & 26,75 & 60,70 & 0,06 \\
& $\pm 14,17$ & $\pm 14,29$ & $\pm 14,17$ & $\pm 42,60$ & $\pm 14,29$ & $\pm 42,60$ & \\
\hline Diafragma & 27,98 & 27,08 & 0,16 & 27,98 & 37,63 & 0,6 & 27,08 & 37,63 & 0,17 \\
& $\pm 17,04$ & $\pm 18,69$ & $\pm 17,04$ & $\pm 29,01$ & & $\pm 18,69$ & $\pm 29,01$ & \\
\hline
\end{tabular}

Protocolo de pesquisa, 2019; Dados são expressos como média \pm desvio padrão; ANOVA $\left(p<0,05^{\star}\right)$; T0 = Avaliação inicial; T2 = Segunda semana de avaliação; T4 = Avaliação final

Na Tabela III, observa-se a evolução das variáveis estudadas após o TMR com aumento significativo entre os três momentos avaliados da $\mathrm{PI}_{\text {máx }}$ do T0 ao T2 $(p=0,005)$, T2 ao T4 $(p=0,01)$ e T0 ao T4, com a média de 66,05 $( \pm 11,65)$ e $99.87( \pm 26,31)$, respectivamente, sugerindo que o treinamento muscular inspiratório foi capaz de incrementar a força muscular respiratória dos sujeitos estudados $(p=0,007)$.

Tabela III - Valores de PImáx, nos T0, T2 e T4, de sete portadores de HTLV-1 participantes do estudo. Belém, Pará, 2019

\begin{tabular}{llllllllll}
\hline Variável & T0 & T2 & $\mathbf{p}$ & T0 & T4 & $\mathbf{p}$ & T2 & T4 & $\mathbf{p}$ \\
\hline Plmáx & 66.05 & 93.25 & 0,005 & 66.05 & 99.87 & 0,007 & 93.25 & 99.87 & 0,01 \\
& $\pm 11,65$ & \pm 19.74 & & \pm 11.65 & \pm 26.31 & & \pm 19.74 & \pm 26.31 & \\
\hline
\end{tabular}

Fonte: Protocolo de pesquisa, 2019; Dados expressos como média \pm desvio padrão; Test $t$ de Student pareado $\left(p<0.05^{\star}\right)$; ANOVA $(p<0.05 \#)$; PImáx = Pressão inspiratória máxima; T0 Avaliação inicial; T2 = Segunda semana de avaliação; T4 = Avaliação final

\section{Discussão}

O estudo teve como referência a análise da EMGs de dois músculos respiratórios (Figura 2), sendo um músculo respiratório principal (diafragma) e o outro acessório (esternocleidomastóideo). O diafragma exerce uma função essencial no processo respiratório e os músculos respiratórios acessórios aumentam a ventilação quando há uma maior solicitação durante o exercício e cooperam com a manutenção da ventilação quando o diafragma é comprometido após doença ou lesão. O músculo diafragma e os músculos esternocleidomastóideos colaboram com graus variados para o processo inspiratório e mostram diferentes funções e características. A coleta do potencial elétrico do diafragma, comparado com os músculos acessórios, não define a gravidade doença [17]. 
O predomínio do sexo feminino no presente estudo (Tabela I) confirma dados de prevalência já apontados na literatura. Esse alto índice é justificado no estudo de Sequeira et al. [18], devido ao início precoce de relações sexuais, múltiplos parceiros sexuais e/ou não uso de preservativos durante a relação sexual, quando esses fatores estão interligados o risco de contaminação é potencializado.

As infecções podem estar relacionadas com a resposta imunológica celular da vagina, na qual essa resposta sujeita-se a ação hormonal modificando, assim, o epitélio e o aumento de linfócitos na região, dessa maneira o agente agressor obtém um ambiente favorável à sua sobrevivência [19,20].

O número de pessoas com a idade maior de 50 anos foi expressivo (Tabela I), sendo este resultado identificado na literatura devido a soroconversão tardia, a soropositividade do vírus aumenta ao longo dos anos [20].

Os pacientes com PET-MAH apresentam várias semelhanças com as lesões evidenciadas na doença Esclerose Múltipla (EM) e como em ambas ocorre desmielinização crônica do sistema nervoso central, pode-se sugerir que portadores sintomáticos com a evolução da doença apresentem comprometimento da musculatura respiratória. Diante desse contexto, destaca-se a diminuição da capacidade de gerar força ou endurance, podendo ocasionar inadequada ventilação pulmonar, especialmente durante o exercício ou na realização de atividades de vida diária (AVD) $[7,21]$.

Verificou-se no estudo durante a primeira avaliação dos sujeitos que os valores de $\mathrm{PI}_{\text {máx }}$ estavam abaixo do previsto. Sendo assim, Levy et al. [22] relatam que os indivíduos com uma doença neurológica crônica, no decorrer do avanço da enfermidade podem apresentar fraqueza dos músculos respiratórios.

O estudo elegeu o treinamento muscular inspiratório (TMI) para esses indivíduos, uma vez que apresentavam os valores de $\mathrm{Pi}_{\text {máx }}$ abaixo do previsto. $\mathrm{O}$ treinamento visou a melhora da função muscular inspiratória, da dispnéia, da força e resistência muscular inspiratória, sendo reconhecidos os benefícios nos resultados pela progressão da $\mathrm{Pi}_{\text {máx }}$ no decurso do TMI $[23,24]$.

Observou-se que no treinamento implementado aos participantes houve uma progressão da força muscular significativa nas três avaliações analisadas (Figura 3). Corroborando Ferreira et al. [25], que constatou efeitos positivos do TMR com pacientes Esclerose Lateral Amiotrófica (ELA) e Esclerose Múltipla, com aumento da $\mathrm{Pi}_{\text {máx, além }}$ da melhora da função ventilatória com aumento do volume expiratório forçado em um segundo (VEF1) no grupo de intervenção em comparação com o controle. Dessa forma, TMR vem sendo indicado com êxito, para pacientes com doenças neurodegenerativas. 
Diante desse contexto, necessita de uma ferramenta para avaliar o desempenho do músculo durante o TMI. Uma das formas de avaliar o comportamento dos músculos respiratórios, suas alterações de amplitude e do espectro de potência da atividade elétrica é através da EMGs, a qual, apresenta-se como um dos poucos métodos de avaliação direta, não invasiva. O EMGs analisa o grau e a duração da atividade muscular e se altera com o treinamento específico para o musculoesquelético. A raiz quadrada da média dos quadrados (RMS) tem sido utilizado para quantificar o sinal eletromiográfico [26].

Analisando a EMGs dos músculos respiratórios, foi verificado um aumento da amplitude de sinal na atividade elétrica muscular em relação a musculatura principal e acessória (Tabela II). Segundo estudo [27] sobre EMGs de músculos respiratórios realizado com candidatos ao transplante de fígado com modificações da força muscular respiratória consequentes ao decréscimo da capacidade oxidativa mitocondrial no tecido muscular, o aumento da atividade muscular advém da exigência de maior força de contração e do aumento da atividade muscular pelo déficit causado pela patologia, desta forma concebendo um aumento da RMS.

O potencial de ação de unidades motoras se caracteriza por vários parâmetros, dentre estes a quantidade de unidades motoras recrutadas, o que reflete enquanto maior um potencial de ação ocorrerá maior recrutamento de fibras musculares esqueléticas para realização do movimento. Verificou-se neste estudo que ocorreu decréscimo do potencial de ação dos dois músculos na primeira metade do treinamento (T0 a T2) (Tabela II), sugerindo que a carga imposta pelo treinamento nas primeiras semanas contribuiu para um melhor desempenho muscular exigindo menos fibras musculares esqueléticas para vencer a resistência imposta [28].

Entretanto, com o decurso do TMI, observou-se o incremento da força muscular e do potencial de ação dos músculos analisados, podendo inferir que com a progressão da carga do treinamento semanal exigia que a musculatura tivesse maior recrutamento de fibras musculares esqueléticas para poder realizar a força estimada (Tabelas II e III). Dessa forma, a literatura constata que o sinal mioelétrico engloba dados sobre a forma do potencial de ação de unidades motoras e assim pode viabilizar uma informação aproximada sobre a eletroneurofisiologia do músculo esquelético ativo e detalhes particulares da atividade muscular a ser estudada [9].

Sendo assim, a força muscular respiratória está fortemente associada ao nível de ativação dos músculos. Constatou-se que a EMGs da musculatura respiratória pode ser utilizada como uma ferramenta para investigar estratégias de recrutamento de unidade motora e como índices indiretos da função muscular, incluindo produção de força muscular e fatigabilidade [29]. 
Além do mais, verificou-se que o músculo esternocleidomastóideo possuiu maior aumento do seu potencial mioelétrico apesar de não ter significância estatística. Isso pode ter ocorrido por diversos fatores dentre eles o tempo de treinamento com a carga estipulada a cada semana ter sido curto e consequentemente não permitiu uma adaptação muscular ou devido ao músculo não estar habituado ao trabalho constante, uma vez que sua contração é exigida quando necessita de uma inspiração forçada. Lembrando que essa musculatura é acessória no processo de respiração, visto que não está ativa durante a respiração basal. Essa musculatura é recrutada e sua contração é ativa em atividades de altas demandas e volumes pulmonares [30].

Costa [31] apontam em seus estudos sobre a relação da eletromiografia quantitativa e o nível de força muscular em pacientes neurológicos, nos quais níveis mais altos de força voluntária produziam maiores potenciais de unidades motoras, os quais foram detectados por agulha e por eletrodo de superfície. Sendo assim, o sinal eletromiográfico constatado com eletrodos de superfície está fortemente relacionado à intensidade contrátil.

A mensuração da força e perda de massa muscular de uma forma não invasiva da musculatura respiratória é uma das limitações da fisioterapia. Como não há trabalhos científicos com a investigação da EMGs em músculos respiratórios diante do HTLV-1, foi necessário recorrer a estudos com pacientes hepatopatas, com doença pulmonar crônica (DPOC) e esclerose múltipla.

A pesquisa trouxe consigo a identificação e um melhor entendimento das alterações nos músculos respiratórios. A EMGs mostrou que pode ser facilmente utilizada no contexto ambulatorial e traz consigo informações significativas da qualidade muscular. As limitações fizeram parte do estudo, tais como a alteração dos sinais pela anatomia do músculo e suas propriedades fisiológicas, por não haver consenso para o posicionamento ideal dos eletrodos de EMGs durante a avaliação não invasiva da ativação do diafragma e a colaboração do paciente com a realização do exame.

Por conseguinte, esta pesquisa abre novas portas para estudos na área, para o melhor atendimento e acompanhamento desses pacientes a fim de que seja otimizada a reabilitação deles, levando uma diminuição nas complicações respiratórias.

\section{Conclusão}

No decurso da proposta ao treinamento muscular ocorreu incremento da força muscular inspiratória com maior recrutamento de unidades motoras dos músculos analisados pela EMGs em voluntários com vírus linfotrópico de célula T humana do tipo 1. 
Referências

1. Schierhout G, McGregor S, Gessain A, Einsiedel L, Martinello M, Kaldor J. Association between HTLV-1 infection and adverse health outcomes: a systematic review and meta-analysis of epidemiological studies. The Lancet Infectious Diseases 2020;20(1):133-43. doi: 10.1016/s1473-3099(19)30402-5 [Crossref]

2. Silva IC, Pinheiro BT, Nobre AFS, Coelho JL, Pereira CCC, Ferreira LSC, et al. Moderate endemicity of the human T-lymphotropic virus infection in the metropolitan region of Belém, Pará, Brazil. Rev Bras Epidemiol 2018;21. doi: 10.1590/1980549720180018 [Crossref]

3. Keikha M, Babaki MKZ, Fonseca LAM, Casseb J. The relevance of HTLV-1-associated myelopathy/tropical spastic paraparesis in Iran: a review study. Reviews in Clinical Medicine 2019;6(2):60-65. doi: 10.22038/RCM.2019.38759.1266 [Crossref]

4. Normando VMF, Dias ÁRN, da Silva ALSE, da Silva DP, de Souza MCS, et al. HTLV-I induces lesions in the pulmonary system: A systematic review. Life Sci 2020;256:117979. doi: 10.1016/j.lfs.2020.117979 [Crossref]

5. Fukuoka J, Tominaga M, Ichikado K, Tanaka T, Ichiyasu H, Kohrogi H, et al. Lung military micro-nodules in human T-cell leukemia virus type I carriers. Pathol Int 2013;108-12. doi: 10.1111/pin.12030 [Crossref]

6. Einsiedel L, Pham H, Wilson K, Walley R, Turpin J, Bangham C, Woodman RJ. Human T-Lymphotropic Virus type 1c subtype proviral loads, chronic lung disease and survival in a prospective cohort of Indigenous Australians. PloS Negl Trop Dis 2018;12(3). doi: 10.1371/journal.pntd.0006281 [Crossref]

7. Falcão LFM. Alterações no sistema pulmonar de pacientes com paraparesia espástica tropical ou mielopatia associada ao HTLV-1 (PET/MAH [Tese]. Ananindeua: Instituto Evandro Chagas; 2016 [Internet]. [cited 2021 mar 15]. Available from: https://patua.iec.gov.br/handle/iec/3125

8. Oliveira AG, De Lima M, Proner J, Guarda GB, Kroth A. A efetividade do treinamento muscular respiratório com Powerbreathe em atletas de basquete. Fisioterapia em Ação 2017 [Internet];21-32. [cited 2021 mar 15]. Available from: https://portalperiodicos.unoesc.edu.br/fisioterapiaemacao/article/view/13262

9. De Luca CJ. The use of surface electromyography in biomechanics. J Appl Biomech 1997;13:135-163. doi: 10.1123/jab.13.2.135 [Crossref]

10. Silva AMO. Eletromiografia de superfície de músculos respiratórios: avaliação de hepatopatas em lista de espera de transplante de fígado e de não hepatopatas [Tese]. São Paulo: Faculdade de Ciências Médicas da Universidade Estadual de Campinas; 2014 [Internet]. [cited 2021 mar 15]. Available from: http://repositorio.unicamp.br/jspui/handle/REPOSIP/312442 
11. Walterspacher S, Pietsch F, Walker DJ, Rocker K, Kabitz HJ. Activation of respiratory muscles during respiratory muscle training. Respir Physiol Neurobiol 2018;247:126-32. doi: 10.1016/j.resp.2017.10.004 [Crossref]

12. Neder JA, Andreoni S, Lerario MC, Nery LE. Reference values for lung function tests. II. Maximal respiratory pressures and voluntary ventilation. Braz J Med Biol Res 1999;32(6):719-27. doi: 10.1590/S0100-879X1999000600007 [Crossref]

13. Merletti $P$, Parker P. Electromyography: physiology, engineering and noninvasive applications. New Jersey: John Wiley \& Son; 2004.

14. Hermens H, Freriks B, Disselhorst-Klug C, Rau G. Development of recommendations for SEMG sensors and sensor placement procedures. J Electromyogr Kinesiol 2000;10(5):361-74. doi: 10.1016/s1050-6411(00)00027-4 [Crossref]

15. Wu W, Guan L, Li X, Lin L,Guo B, Yang Y, et al. Correlation and compatibility between surface respiratory electromyography and transesophageal diaphragmatic electromyography measurements during treadmill exercise in stable patients with COPD. Int J Chron Obstruct Pulmon Dis 2017;6(12):3273-80. doi: 10.2147/COPD.S148980 [Crossref]

16. Ray AD, Udhoji S, Mashtare TL, Fisher NM. A combined inspiratory and expiratory muscle training program improves respiratory muscle strength and fatigue in multiple sclerosis. Arch Phys Med Rehabil 2013;94(10):1964-70. doi:

10.1016/j.apmr.2013.05.005 [Crossref]

17. Zhang DD, Gan L, Zhu XF, Zhag LL, Gao J, Shi LC, et al. Neural respiratory drive measured using surface electromyography of diaphragm as a physiological biomarker to predict hospitalization of acute exacerbation of chronic obstructive pulmonary disease patients. Chin Med J 2018;131(23):2800-7. doi: 10.4103/0366-6999.246057 [Crossref]

18. Sequeira CG, Tamegão-Lopes BP, Santos EJ, Ventura AM, Moraes-Pinto MI, Succi $R C$. Descriptive study of HTLV infection in a population of pregnant women from the state of Pará, Northern Brazil. Rev Soc Bras Med Trop 2012;45(4):453-6. doi: 10.1590/S0037-86822012005000007 [Crossref]

19. Lucena AAS, Michelin MA, Guimarães MVMB, Lodi CTC, Miranda MIL, Murta EFC, et al. Resposta imune celular ao papilomavírus humano em mulheres infectadas e não infectadas pelo vírus da imunodeficiência humana. Femina 2011 [Internet];39(3):150-5. [cited 2021 mar 15]. Available from: http://files.bvs.br/upload/S/01007254/2011/v39n3/a2500.pdf

20. Glória LM, Damasceno SA, Rodrigues LR, Santos MSB, Medeiros R, Dias GAS, et al.. Perfil clínicoepidemiológico de pacientes infectados pelo HTLV-1 em Belém/Pará. Cad Saúde Coletiva 2015;23(2):157-62. doi: 10.1590/1414-462X201400050087 [Crossref]

21. Morais MT, Caires SS. Perfil socioepidemiológico dos portadores do HTLV em um município do sudoeste baiano. Revista de Saúde Coletivada UEFS 2017;7(2):18-21. doi: 10.13102/rscdauefs.v0i0.1220 [Crossref] 
22. Levy J, Prigent H, Bensmail D. Respiratory rehabilitation in multiple sclerosis: A narrative review of rehabilitation techniques. Ann Phys Rehabil Med 2018;61:38-45. doi: 10.1016/j.rehab.2017.06.002 [Crossref]

23. Kock KS, Caiônico JC, Luiz AR, Arent YA, Fernandes I. Análise da pressão inspiratória com alto e baixo fluxos em resistor alinear. ASSOBRAFIR Ciência 2015;6(1):13-20. doi: 10.47066/2177-9333/ac.18983 [Crossref]

24. Tzelepis GE, McCool FD. Respiratory dysfunction in multiple sclerosis. Respir Med 2015;09(6):671-79. doi: 10.1016/j.rmed.2015.01.018 [Crossref]

25. Ferreira GD, Costa AC, Plentz RD, Coronel CC, Sbruzzi G. Respiratory training improved ventilatory function and respiratory muscle strength in patients with multiple sclerosis and lateral amyotrophic sclerosis: systematic review and meta-analysis. Physiotherapy 2016;102(3):221-28. doi: 10.1016/j.physio.2016.01.002 [Crossref]

26. Reis IMM, Ohara DG, Januário LB, Basso-Vanelli RP, Oliveira AB, Jamami M. Surface electromyography in inspiratory muscles in adults and elderly individuals: A systematic review. J Electromyogr Kinesiol 2019;44:139-55. doi: 10.1016/j.jelekin.2019.01.002 [Crossref]

27. Custódio SR, Santos RB, Silva ÁMO, Ratti LDSR, Boin IDFSF, Duarte RP, et al. Effects of respiratory endurance test detected by surface electromyography (sEMG) in patients candidates for liver transplantation. J Bras Transpl 2016 [Internet];19(4):6-10. [cited 2021 may 14]. Available from: https://site.abto.org.br/wp-content/uploads/2020/06/4 21. pdf

28. Duarte RP, Sentanin AC, Silva AMO, Tonella RM, Duarte GL, Ratti LSR, Boin IFSF. Diaphragm muscle surface electromyography in patients submitted to liver transplant and eligible for extubation. Transplantation Proceedings 2017;49(4),829-31. doi: 10.1016/j.transproceed.2017.01.059 [Crossref]

29. Lozano-García M, Sarlabous L, Moxham J, Rafferty GF, Torres A, Jané R, Jolley C. Surface mechanomyography and electromyography provide noninvasive indices of inspiratory muscle force and activation in healthy subjects. Sci Rep 2018;8:1-13. doi: 10.1038/s41598-018-35024-z [Crossref]

30. Grosu HB, Ost DE, Lee YI, Song J, Li L, Eden, E, Rose K. Diaphragm muscle thinning in subjects receiving mechanical ventilation and its effect on extubation. Resp Care 2017;62(7):904-11. doi: 10.4187/respcare.05370 [Crossref]

31. Costa HLLS. Análise da atividade dos músculos respiratórios por meio da eletromiografia de superfície durante a mensuração do índice de esforço inspiratório cronometrado [Dissertação]. Niteroi/RJ: UFF;2019 [Internet]. [cited 2020 dez 17]. Available from: https://app.uff.br/riuff/handle/1/13089 